\title{
Resummation prediction on Higgs and vector boson associated production with a jet veto at the LHC
}

\author{
Ding Yu Shao, ${ }^{a}$ Chong Sheng $\mathbf{L i}^{a, b}$ and Hai Tao $\mathbf{L i}^{a}$ \\ ${ }^{a}$ School of Physics and State Key Laboratory of Nuclear Physics and Technology, \\ Peking University, Beijing 100871, China \\ ${ }^{b}$ Center for High Energy Physics, \\ Peking University, Beijing 100871, China \\ E-mail: shaodingyu@pku.edu.cn, csli@pku.edu.cn, lihaitao@pku.edu.cn
}

ABSTRACT: We investigate the resummation effects for the SM Higgs and vector boson associated production at the LHC with a jet veto in soft-collinear effective theory using "collinear anomalous" formalism. We calculate the jet vetoed invariant mass distribution and the cross section for this process at Next-to-Next-to-Leading-Logarithmic level, which are matched to the QCD Next-to-Leading Order results, and compare the differences of the resummation effects with different jet veto $p_{T}^{\text {veto }}$ and jet radius $R$. Our results show that both resummation enhancement effects and the scale uncertainties decrease with the increasing of jet veto $p_{T}^{\text {veto }}$ and jet radius $R$, respectively. When $p_{T}^{\text {veto }}=25 \mathrm{GeV}$ and $R=0.4(0.5)$, the resummation effects reduce the scale uncertainties of the Next-to-Leading Order jet vetoed cross sections to about $7 \%$ (6\%), which lead to increased confidence on the theoretical predictions. Besides, after including resummation effects, the PDF uncertainties of jet vetoed cross section are about $7 \%$.

Keywords: Resummation, Renormalization Group, QCD, Higgs Physics

ARXIV EPRINT: 1309.5015 


\section{Contents}

1 Introduction 1

2 Factorization in SCET $\quad 3$

3 Hard function and beam function $\quad 5$

3.1 Hard function 5

3.2 Beam function $\quad 6$

3.3 RG improved cross section $\quad 8$

4 Numerical results $\quad 9$

$\begin{array}{llr}4.1 & \text { Leading singular jet vetoed cross section } & 9\end{array}$

$\begin{array}{ll}4.2 \text { Scale uncertainties } & 10\end{array}$

4.3 RG improved phenomenology predictions at the LHC 12

$\begin{array}{llr}5 & \text { Conclusion } & 15\end{array}$

$\begin{array}{ll}\text { A Calculation of beam functions } & 16\end{array}$

\section{Introduction}

Recently, both the ATLAS [1] and CMS [2] collaborations at the CERN Large Hadron Collider (LHC) have found a Standard Model (SM) Higgs boson particle with a mass around $125 \mathrm{GeV}$ mainly through gluon-gluon fusion channel. However, by means of modern jet substructure methods, the associated production of Higgs boson $H$ and vector boson $V\left(V=Z, W^{ \pm}\right)$is also an important process to study the Higgs boson at the LHC.

The efforts of obtaining accurate theoretical predictions for $H V$ associated production at the hadron colliders have been for a long time. The Next-to-Leading-Order (NLO) QCD and Electro-Weak (EW) corrections have been performed in refs. [3-7]. Besides, the QCD Next-to-Next-to-Leading-Order (NNLO) corrections of the total inclusive cross section for $H V$ associated production were calculated in refs. [8-10]. The corresponding numerical results have been implemented in numerical code VH@NNLO [11], which is now available on the website. Recently, in ref. [12] the NNLO QCD corrections of exclusive cross section for $H W^{ \pm}$associated production were completed based on the transverse momentum substraction formalism [13]. And the effects of NLO QCD corrections to both $H W^{ \pm}$associated production and subsequent decay of $H \rightarrow b \bar{b}$ were investigated in ref. [14]. However, the completely NNLO QCD corrections for both $H V$ associated production and subsequent decay of $H \rightarrow b \bar{b}$ are still absent so far.

The process for Higgs boson production involve a number of jets associated radiation at hadron colliders. The Standard Model (SM) backgrounds process produce the similar 
signature with additional energetic jets. For example, the $H W^{ \pm}$associated production with Higgs decaying to $b \bar{b}$ has large QCD backgrounds at hadron colliders. When leptonic decay modes of $W^{ \pm}$is considered, the semi-leptonic decays of $t \bar{t}$ can become a significant irreducible background. Due to the fact that the SM top quark pair production has more hard jets from decay of top quark than the $H W^{ \pm}$process, a jet veto can be used to suppress $t \bar{t}$ background [15]. Thus, a veto on the additional undesired jets $p_{T}^{\text {jet }}<p_{T}^{\text {veto }}$ is needed to distinguish the signal and background process, and improve the significance of $H W^{ \pm}$production.

Due to the presence of the jet veto $p_{T}^{\text {veto }}$, a small energy scale $p_{T}^{\text {veto }}$ is introduced into the physical process, which is about $20 \sim 30 \mathrm{GeV}$. Therefore there exist large logarithmic terms $\ln ^{n} p_{T}^{\text {veto }} / Q$ in the perturbative calculations at the all order where $Q$ denotes the hard scale in the process, and these large logarithms need be resummed for improving the accuracy of the theoretical predictions. By means of parton showers, the Leading-Logarithmic (LL) predictions on the cross section with a jet veto are available $[16,17]$. Besides, the event shape variables of beam thrust, $N$-jettiness and $E_{T}=\sum\left|\vec{p}_{T}\right|$ are used to implement a jet veto on additional emissions [18-23]. In the last year the jet veto efficiency in Higgs boson and Drell-Yan production at the hadron collider at the NLL level has been investigated with the CAESAR approach [24] in ref. [25]. After that the all order factorization formula for single Higgs boson production with a jet veto $p_{T}^{\text {veto }}$ have been firstly derived at the leading power of $\lambda=p_{T}^{\text {veto }} / m_{H}$ with the soft-collinear effective theory (SCET) [26-28] based on "collinear anomaly" formalism [29], and the large double logarithmic terms have been resummed to NNLL order in ref. [30]. Then in ref. [31], the results of ref. [24] combining the Drell-Yan like boson transverse momentum resummations [29, 32-34] are used to obtain NNLL resummed jet veto efficiencies for Higgs boson and Drell-Yan production at hadron colliders. Very recently, the $\mathrm{N}^{2} \mathrm{LL}^{\prime}+\mathrm{NNLO}$ predictions on the jet veto cross section for single Higgs boson production have been investigated in ref. [35, 36]. In ref. [35] the anomaly coefficient $d_{2}^{\text {veto }}(R)$ was firstly calculated using the SCET and the two loop low energy matrix elements are extracted numerically. The main theoretical approximation comes from the lack of the anomaly coefficient $d_{3}^{\text {veto }}(R)$ and the four loop cusp anomalous dimension. And in ref. [36] the "rapidity renormalization group" formalism [37, 38] are used, where the NNLO soft function and the NNLO beam function are partly derived. The remaining contributions are numerically extracted. The main approximation also comes from unknown higher-order anomalous dimensions.

In this paper we investigate the resummation effects in $H V$ associated production at the hadron collider with a jet veto using SCET based on the "collinear anomaly" formalism. We firstly calculate the Higgs and vector boson invariant mass distribution and the total cross section with a jet veto at the NNLL level, which are matched to the QCD NLO results. Nevertheless, the jet veto efficiency for $H V$ associated production have be approximated studied in ref. [15], where the jet veto cross section is defined as

$$
\sigma\left(p_{T, H V}\right)=\int_{0}^{p_{T, H V}} d p_{T, H V} \frac{d \sigma}{d p_{T, H V}}
$$

Here $p_{T, H V}$ is the transverse momentum of $H V$ and $d \sigma / d p_{T, H V}$ is NLL+NLO $H V$ trans- 
verse momentum distribution. However the logarithmic terms at small $p_{T, H V}$ are different from those induced by jet veto $p_{T}^{\text {veto }}$ at the NNLL level, so those studies in ref. [15] only give a qualitative analysis.

The arrangement of this paper is as follows. In section 2 we derive the factorization formula for $H V$ associated production with a jet veto at the hadron collider. In section 3 we calculate the hard and beam matching coefficients at the NLO, and present Renormalization Group (RG) improved differential cross section analytically. In section 4 we discuss the numerical results of cross section and the invariant mass distribution with a jet veto. We conclude in section 5 .

\section{Factorization in SCET}

In this section we describe the derivation of factorization for $H V$ associated production with a jet veto in SCET based on the "collinear anomaly" formalism. In ref. [15] the threshold resummation of the total cross section and invariant mass distribution for $H \mathrm{~V}$ associated production in SCET has been investigated. However, the resummation for $H V$ associated production with a jet veto discussed in this paper is genuinely different from threshold resummation. We consider the process of stable Higgs and vector boson associated production,

$$
N_{1}\left(P_{1}\right)+N_{2}\left(P_{2}\right) \rightarrow H\left(p_{3}\right)+V\left(p_{4}\right)+X^{\prime}\left(p_{X}\right),
$$

where $X^{\prime}$ is the final hadronic state passing jet veto $p_{T}^{\text {veto }}$. In the Born approximation $H V$ associated production is mainly induced by quark anti-quark annihilation,

$$
q\left(p_{1}\right)+\bar{q}\left(p_{2}\right) \rightarrow H\left(p_{3}\right)+V\left(p_{4}\right),
$$

where $p_{1}=x_{1} P_{1}$ and $p_{2}=x_{2} P_{2}$. We define the kinematic invariants,

$$
s=\left(P_{1}+P_{2}\right)^{2}, \quad \hat{s}=\left(p_{1}+p_{2}\right)^{2}, \quad M^{2}=\left(p_{3}+p_{4}\right)^{2} .
$$

In the presence of a jet veto $p_{T}^{\text {veto }}$, the kinematic region we are interested in is

$$
\hat{s}, M^{2}, m_{H}^{2}, m_{V}^{2} \gg\left(p_{T}^{\text {veto }}\right)^{2} \gg \Lambda_{\mathrm{QCD}}^{2}
$$

It is convenient to introduce two light-like reference vectors $n=(1,0,0,1)$ and $\bar{n}=$ $(1,0,0,-1)$ along the beam axis and any four vector can be decomposed as

$$
p^{\mu}=n \cdot p \frac{\bar{n}^{\mu}}{2}+\bar{n} \cdot p \frac{n^{\mu}}{2}+p_{\perp}^{\mu} \equiv p_{+}^{\mu}+p_{-}^{\mu}+p_{\perp}^{\mu} .
$$

Hence momentum $p^{\mu}$ can be denoted by $p^{\mu}=\left(p^{+}, p^{-}, p_{\perp}\right)$. Different momentum modes relevant to our discussions are collinear mode $p_{n}^{\mu} \sim M\left(\lambda^{2}, 1, \lambda\right)$, anti-collinear mode $p_{\bar{n}}^{\mu} \sim$ $M\left(1, \lambda^{2}, \lambda\right)$ and soft mode $p_{s}^{\mu} \sim M(\lambda, \lambda, \lambda)$. Here $\lambda=p_{T}^{\text {veto }} / M$ is treated as a small expansion parameter. In order to handle these momentum regions, SCET is a very useful framework, which is very suitable to deal with the scattering processes with multiple scales. 
For the Drell-Yan like process the chiral current operator for initial quark and antiquark can be written as

$$
J^{\mu}=g_{L}^{V} \bar{q}_{i} \gamma^{\mu} P_{L} q_{j}+g_{R}^{V} \bar{q}_{i} \gamma^{\mu} P_{R} q_{j}
$$

where the $i, j$ subscripts represent the flavors of quark and the couplings $g_{L(R)}^{V}$ for $W$ and $Z$ boson are separately

- $g_{L}^{W}=\frac{V_{i j}}{\sqrt{2} S_{w}}, \quad g_{R}^{W}=0$,

- $g_{L}^{Z}=\frac{I_{f}^{3}-S_{w}^{2} Q_{f}}{S_{w} C_{w}} \delta_{i j}, \quad g_{R}^{Z}=-\frac{S_{w}}{C_{w}} Q_{f} \delta_{i j}$,

where $V_{i j}$ is the CKM matrix, $I_{f}^{3}$ is the third component of isospin and $Q_{f}$ is the electric charge for quark. Here $S_{w}=\sin \theta_{w}$ and $C_{w}=\cos \theta_{W}$, where $\theta_{W}$ is Weinberg angle. At the leading power of $\lambda$, the chiral current operators are matched onto SCET operators as

$$
J^{\mu} \rightarrow C_{V}\left(-q^{2}-i \epsilon, \mu^{2}\right)\left(g_{L}^{V} \bar{\chi}_{\bar{n}} S_{\bar{n}}^{\dagger} \gamma^{\mu} P_{L} S_{n} \chi_{n}+g_{R}^{V} \bar{\chi}_{\bar{n}} S_{\bar{n}}^{\dagger} \gamma^{\mu} P_{R} S_{n} \chi_{n}\right) .
$$

Here $C_{V}$ is the hard matching coefficient and $\bar{\chi}_{n(\bar{n})}$ are the gauge invariant combinations of (anti-)collinear quark fields and Wilson lines in SCET. The soft degrees of freedom are contained in the soft Wilson lines $S_{n(\bar{n})}$.

In order to define the jets at the hadron collider, the sequential recombination jet algorithms are used [39]. The longitudinal boost invariant distance measures $d_{i j}$ and $d_{i B}$ are defined by

$$
\begin{aligned}
d_{i j} & =\min \left(p_{T i}^{n}, p_{T j}^{n}\right) \Delta R_{i j} / R, \quad \Delta R_{i j}=\sqrt{\left(y_{i}-y_{j}\right)^{2}+\left(\phi_{i}-\phi_{j}^{2}\right)}, \\
d_{i B} & =p_{T i}^{n},
\end{aligned}
$$

where $R$ is the jet radius parameter. Here $n=-1,0$ and 1 represent the inclusive anti$k_{T}$ [40, 41], Cambridge-Aachen [42, 43] and $k_{T}$ [44] jet algorithms, respectively. As is shown in ref. [30], the different momentum modes (collinear, anti-collinear and soft) can not be grouped into the same jet after performing jet algorithms as long as jet radius parameter satisfies

$$
\lambda \ll R \ll \ln \lambda,
$$

where $R \sim \mathcal{O}(1)$ is assumed. Therefore the jet veto can be applied in collinear, anti-collinear and soft region, respectively. After factorizing the contributions from hard, collinear, anticollinear, and soft degrees of freedom in the SCET, we can obtain the factorized differential cross section for the rapidity $Y$ and the invariant mass $M$ of Higgs and vector boson at the leading power of $\lambda$

$$
\begin{aligned}
\frac{d \sigma\left(p_{T}^{\text {veto }}\right)}{d M^{2} d Y}= & \frac{\sigma_{0}}{s} \mathcal{H}\left(M^{2}, \mu^{2}\right) \mathcal{B}_{q / N_{1}}^{n}\left(\zeta_{1}, p_{T}^{\text {veto }}, \mu\right) \mathcal{B}_{\bar{q} / N_{2}}^{\bar{n}}\left(\zeta_{2}, p_{T}^{\text {veto }}, \mu\right) \mathcal{S}\left(p_{T}^{\text {veto }}, \mu\right) \\
& +(q \leftrightarrow \bar{q}),
\end{aligned}
$$


where $\zeta_{1,2}=(M / \sqrt{s}) e^{ \pm Y}$ and $\sigma_{0}$ is the LO total cross section, and it is defined as

$$
\sigma_{0}=\frac{G_{F}^{2} S_{w}^{4} m_{W}^{4}}{36 \pi M^{2}} g_{V V H}^{2}\left(g_{L}^{2}+g_{R}^{2}\right) \Lambda^{1 / 2}\left(m_{V}^{2}, m_{H}^{2}, M^{2}\right) \frac{\Lambda\left(m_{V}^{2}, m_{H}^{2}, M^{2}\right)+12 m_{V}^{2} / M^{2}}{\left(1-m_{V}^{2} / M^{2}\right)^{2}},
$$

with

$$
\Lambda(x, y, z)=(1-x / z-y / z)^{2}-4 x y / z^{2} .
$$

Here $m_{V}$ is the mass of vector boson, $G_{F}$ is Fermi constant, $g_{V V H}$ is the coupling between Higgs and vector boson, $g_{W W H}=1 / S_{w}$ and $g_{Z Z H}=1 /\left(S_{w} C_{w}\right)$. In the eq. (2.11), the hard function $\mathcal{H}$ is the absolute value squared of the hard matching coefficient $\mathcal{H}\left(M^{2}, \mu^{2}\right)=$ $\left|C_{V}\left(-M^{2}-i \epsilon, \mu^{2}\right)\right|^{2}$, and the collinear matrix elements $\mathcal{B}_{q / N}^{n}$ correspond to the PDFs, which are defined as [35]

$$
\begin{aligned}
\mathcal{B}_{q / N}^{n}\left(z, p_{T}^{\text {veto }}, \mu\right)=\int \frac{d t}{2 \pi} e^{-i z t \bar{n} \cdot p} \sum_{X_{n}, \text { reg }} \mathcal{M}_{\text {veto }}\left(p_{T}^{\text {veto }}, R,\left\{\underline{p_{n}}\right\}\right) \\
\times\left\langle N(p)\left|\bar{\chi}_{n}(t \bar{n})\right| X_{n}\right\rangle\left\langle X_{n}\left|\chi_{n}(0)\right| N(p)\right\rangle .
\end{aligned}
$$

Here the summation over the collinear states $X_{n}$ is constrained by the jet veto, and the corresponding constraints are included in the function $\mathcal{M}_{\text {veto }}$, which depends on the collinear momentums $\left\{\underline{p_{n}}\right\}$. Similarly, the soft function is defined in terms of the vacuum matrix element of the product for the soft Wilson lines constrained by the jet veto as [35]

$$
\mathcal{S}\left(p_{T}^{\mathrm{veto}}, \mu\right)=\frac{1}{N_{c}} \sum_{X_{s}, \text { reg }} \mathcal{M}_{\text {veto }}\left(p_{T}^{\text {veto }}, R,\left\{\underline{p_{s}}\right\}\right)\left\langle 0\left|\left[S_{n}^{\dagger} S_{\bar{n}}\right](0)\right| X_{s}\right\rangle\left\langle X_{s}\left|\left[S_{\bar{n}}^{\dagger} S_{n}\right](0)\right| 0\right\rangle .
$$

The definitions of the (anti-)collinear and soft functions involve light-cone singularities which are not regularized by dimensional regularization. These divergences can be regularized in various ways $[29,37,45,46]$, and the product of the (anti-)collinear and soft functions are free from the light-cone singularities. However, anomalous dependence on the hard scale $M$ remains, which was called "collinear anomaly" [29].

\section{Hard function and beam function}

\subsection{Hard function}

The hard matching coefficient $C_{V}\left(-M^{2}, \mu_{h}^{2}\right)$ (here and below the negative arguments are understood with a $-i \epsilon$ prescription) can be obtained by matching the two quark operators in the full theory onto the operator in SCET, where the infrared divergences are subtracted in the $\overline{\mathrm{MS}}$ scheme. The two loop results for the $C_{V}\left(-M^{2}, \mu_{h}^{2}\right)$ have been available in ref. [47]. Up to NLO, it can be written as

$$
C_{V}\left(-M^{2}, \mu_{h}^{2}\right)=1+\frac{C_{F} \alpha_{s}\left(\mu_{h}^{2}\right)}{4 \pi}\left(-L_{H}^{2}+3 L_{H}-8+\frac{\pi^{2}}{6}\right)
$$


where $L_{H}=\ln \left(-M^{2} / \mu_{h}^{2}\right)$. The RG equation for $C_{V}\left(-M^{2}, \mu^{2}\right)$ is governed by the anomalous-dimension, the structure of which has been predicted up to four-loop level for the case of massless partons [48]. The $C_{V}\left(-M^{2}, \mu^{2}\right)$ satisfies the RG equation

$$
\frac{d}{d \ln \mu} C_{V}\left(-M^{2}, \mu^{2}\right)=\left[\Gamma_{\text {cusp }}^{F}\left(\alpha_{s}\right) \ln \frac{-M^{2}}{\mu^{2}}+\gamma^{V}\left(\alpha_{s}\right)\right] C_{V}\left(-M^{2}, \mu^{2}\right),
$$

where $\Gamma_{\text {cusp }}^{F}\left(\alpha_{s}\right)$ is the cusp anomalous dimension, while $\gamma^{V}\left(\alpha_{s}\right)$ controls the singlelogarithmic evolution. After solving the RG equation, we have the hard matching coefficient

$$
C_{V}\left(-M^{2}, \mu_{f}^{2}\right)=\exp \left[2 S\left(\mu_{h}^{2}, \mu_{f}^{2}\right)-a_{\Gamma}\left(\mu_{h}^{2}, \mu_{f}^{2}\right) \ln \frac{-M^{2}}{\mu_{h}^{2}}-a_{\gamma^{V}}\left(\mu_{h}^{2}, \mu_{f}^{2}\right)\right] C_{V}\left(-M^{2}, \mu_{h}^{2}\right),
$$

where $S\left(\nu^{2}, \mu^{2}\right)$ and $a_{\Gamma}\left(\nu^{2}, \mu^{2}\right)$ are defined as

$$
\begin{aligned}
S\left(\nu^{2}, \mu^{2}\right) & =-\int_{\alpha_{s}\left(\nu^{2}\right)}^{\alpha_{s}\left(\mu^{2}\right)} d \alpha \frac{\Gamma_{\text {cusp }}^{F}(\alpha)}{\beta(\alpha)} \int_{\alpha_{s}\left(\nu^{2}\right)}^{\alpha} \frac{d \alpha^{\prime}}{\beta\left(\alpha^{\prime}\right)}, \\
a_{\Gamma}\left(\nu^{2}, \mu^{2}\right) & =-\int_{\alpha_{s}\left(\nu^{2}\right)}^{\alpha_{s}\left(\mu^{2}\right)} d \alpha \frac{\Gamma_{\text {cusp }}^{F}(\alpha)}{\beta(\alpha)} .
\end{aligned}
$$

$a_{\gamma^{V}}$ has a similar expression. Finally, the hard function is given by

$$
\mathcal{H}\left(M^{2}, \mu_{f}^{2}\right)=\left|C_{V}\left(-M^{2}, \mu_{f}^{2}\right)\right|^{2} .
$$

Up to NNLL level, we need three loop cusp anomalous dimension and two loop normal anomalous dimension, and their explicit expressions are collected in the appendices of ref. [47].

\subsection{Beam function}

In ref. [18] a first study on the factorization theorem with beam function is performed. At hadron colliders if there exists experimental restrictions, which introduce a new kinematic scale on the hadronic final states, then the factorization does not yield standard PDFs for the initial states. Thus beam function is necessary to properly describe the jets from initial states.

The collinear matrix element $\mathcal{B}_{q / N}^{n}\left(z, p_{T}^{\text {veto }}, \mu\right)$ defined in eq. (2.14) are intrinsically nonperturbative objects. In the limit $p_{T}^{\text {veto }} \gg \Lambda_{\mathrm{QCD}}$, they can be matched onto the standard Parton Distribution Functions (PDFs) via [30]

$$
\mathcal{B}_{q / N}^{n}\left(\zeta, p_{T}^{\text {veto }}, \mu\right)=\sum_{i=g, q, \bar{q}} \int_{\zeta}^{1} \frac{d z}{z} \mathcal{I}_{q \leftarrow i}\left(z, p_{T}^{\text {veto }}, \mu\right) f_{i / N}(\zeta / z, \mu),
$$

where the beam function $\mathcal{I}_{q \leftarrow i}\left(z, p_{T}^{\text {veto }}, \mu\right)$ can be calculated up to QCD NLO and we collect those results in appendix A for the convenience. The product of initial state beam functions 
can be factorized as

$$
\begin{aligned}
& {\left[\mathcal{I}_{q \leftarrow i}\left(z_{1}, p_{T}^{\text {veto }}, \mu_{f}\right) \mathcal{I}_{\bar{q} \leftarrow j}\left(z_{2}, p_{T}^{\text {veto }}, \mu_{f}\right)\right]_{q^{2}=M^{2}}=} \\
& \left(\frac{M}{p_{T}^{\text {veto }}}\right)^{-2 F_{q \bar{q}}\left(p_{T}^{\text {veto }}, \mu_{f}\right)} I_{q \leftarrow i}\left(z_{1}, p_{T}^{\text {veto }}, \mu_{f}\right) I_{\bar{q} \leftarrow j}\left(z_{2}, p_{T}^{\text {veto }}, \mu_{f}\right),
\end{aligned}
$$

where the anomalous dependence on $M$ is factorized out and is controlled by the function $F_{q \bar{q}}$, while the function $I_{q \leftarrow i}$ is independent on the hard scale $M$. The RG equation for $F_{q \bar{q}}$ can be written as

$$
\frac{d}{d \ln \mu} F_{q \bar{q}}\left(p_{T}^{\text {veto }}, \mu\right)=2 \Gamma_{\text {cusp }}^{F}\left(\alpha_{s}\right) .
$$

After solving this RG equation, we can obtain $F_{q \bar{q}}$ up to two loop as

$$
F_{q \bar{q}}\left(p_{T}^{\text {veto }}, \mu_{f}\right)=a_{s}\left[\Gamma_{0}^{F} L_{\perp}+d_{1}^{\mathrm{veto}}(R)\right]+a_{s}^{2}\left[\Gamma_{0}^{\mathrm{F}} \beta_{0} \frac{L_{\perp}^{2}}{2}+\Gamma_{1}^{\mathrm{F}} L_{\perp}+d_{2}^{\mathrm{veto}}(R)\right],
$$

where the anomaly coefficient $d_{i}^{\text {veto }}(R)$ can be extracted from fixed order calculations of beam function. In order to cancel large logarithms dependence in function $I_{q \leftarrow i}$, the double logarithmic terms in the $I_{q \leftarrow i}$ functions are exponentiated via

$$
\bar{I}_{q \leftarrow i}\left(z, p_{T}^{\text {veto }}, \mu_{f}\right)=e^{-h_{F}\left(p_{T}^{\text {veto }}, \mu_{f}\right)} I_{q \leftarrow i}\left(z, p_{T}^{\text {veto }}, \mu_{f}\right),
$$

where the $\mathrm{RG}$ equation for $h_{F}$ can be written as

$$
\frac{d}{d \ln \mu} h_{F}\left(p_{T}^{\text {veto }}, \mu\right)=2 \Gamma_{\text {cusp }}^{F}\left(\alpha_{s}\right) \ln \frac{\mu}{p_{T}^{\text {veto }}}-2 \gamma^{q}\left(\alpha_{s}\right) .
$$

Here $\gamma^{q}$ is the anomalous dimension of collinear quark field. The solution of this RG equation for $h_{F}$ is given by

$$
h_{F}\left(p_{T}^{\text {veto }}, \mu_{f}\right)=a_{s}\left(\Gamma_{0}^{F} \frac{L_{\perp}^{2}}{4}-\gamma_{0}^{q} L_{\perp}\right),
$$

where the normalization condition of $h_{F}\left(p_{T}^{\text {veto }}, p_{T}^{\text {veto }}\right) \equiv 0$ is chosen. Now, the RG equation for the matching function $\bar{I}_{q \leftarrow i}\left(z, p_{T}^{\text {veto }}, \mu_{f}\right)$ can be written as

$$
\frac{d}{d \ln \mu} \bar{I}_{q \leftarrow i}\left(z, p_{T}^{\text {veto }}, \mu\right)=-\sum_{j} \int_{z}^{1} \frac{d \zeta}{\zeta} \bar{I}_{q \leftarrow j}\left(\zeta, p_{T}^{\text {veto }}, \mu\right) \mathcal{P}_{j \leftarrow i}\left(z / \zeta, \alpha_{s}\right) .
$$

Here $\mathcal{P}_{j \leftarrow i}$ are the DGLAP splitting functions. Obviously, the new functions $\bar{I}_{q \leftarrow j}$ evolve exactly following the DGLAP equations with an opposite sign. Solving the RG equation (3.14), up to the NLO, we have

$$
\bar{I}_{q \leftarrow i}\left(z, p_{T}^{\text {veto }}, \mu_{f}\right)=\delta(1-z) \delta_{q i}+a_{s}\left[-\mathcal{P}_{q \leftarrow i}^{(1)}(z) \frac{L_{\perp}}{2}+\mathcal{R}_{q \leftarrow i}(z)\right] .
$$


Here we define $a_{s} \equiv \alpha_{s} /(4 \pi), L_{\perp} \equiv 2 \ln \left(\mu_{f} / p_{T}^{\text {veto }}\right)$. After calculating complete one loop function $\mathcal{I}_{q \leftarrow i}\left(z, p_{T}^{\text {veto }}, \mu\right)$, we have

$$
\begin{aligned}
& d_{1}^{\text {veto }}(R)=0, \\
& \mathcal{R}_{q \leftarrow q}(z)=C_{F}\left[2(1-z)-\frac{\pi^{2}}{6} \delta(1-z)\right], \\
& \mathcal{R}_{q \leftarrow g}(z)=4 T_{F} z(1-z) .
\end{aligned}
$$

The two loop coefficient $d_{2}^{\mathrm{veto}}(R)$ expanded as small $R$ has been analytically calculated in ref. [35], and it has the form

$$
d_{2}^{\mathrm{veto}}=d_{2}^{q}-8 \Gamma_{0}^{\mathrm{F}} f(R),
$$

where $d_{2}^{q}$ is the corresponding coefficient in the small transverse momentum resummation for Drell-Yan process and is given by

$$
d_{2}^{q}=\Gamma_{0}^{F}\left[\left(\frac{202}{27}-7 \zeta_{3}\right) C_{A}-\frac{56}{27} T_{F} n_{f}\right],
$$

and the function $f(R)$ can also be numerically extracted from ref. [24, 31], which agrees well with the analytical expression in ref. [35], which is

$$
\begin{aligned}
f(R)= & -\left(1.09626 C_{A}+0.1768 n_{f} T_{F}\right) \ln R+\left(0.6072 C_{A}-0.0308 T_{F} n_{f}\right) \\
& +\left(0.2639 C_{A}-0.8225 C_{F}+0.02207 T_{F} n_{f}\right) R^{2} \\
& -\left(0.0226 C_{A}-0.0625 C_{F}+0.0004 T_{F} n_{f}\right) R^{4}+\cdots
\end{aligned}
$$

\subsection{RG improved cross section}

Based on the regularization scheme in ref. [45], the soft function $\mathcal{S}\left(p_{T}^{\text {veto }}, \mu\right) \equiv 1$ to all order because the integrals of soft function are scaleless in the high order perturbative calculations. Therefore, after integrating the the rapidity variable $Y$, we finally have the resummed cross section

$$
\frac{d \sigma\left(p_{T}^{\text {veto }}\right)}{d M^{2}}=\frac{\sigma_{0}}{s} \bar{H}\left(M, p_{T}^{\text {veto }}\right) \int_{\tau}^{1} \frac{d z}{z} \overline{\mathbb{I}}_{i j}\left(z, p_{T}^{\text {veto }}, \mu_{f}\right) f f_{i j}\left(\frac{\tau}{z}, \mu_{f}\right) .
$$

where we have defined the RG invariant hard function as

$$
\bar{H}\left(M, p_{T}^{\text {veto }}\right)=\mathcal{H}\left(M^{2}, \mu_{f}^{2}\right)\left(\frac{M}{p_{T}^{\text {veto }}}\right)^{-2 F_{q \bar{q}}\left(p_{T}^{\text {veto }}, \mu_{f}\right)} e^{2 h_{F}\left(p_{T}^{\text {veto }}, \mu_{f}\right)},
$$

and the convolutions of $\bar{I}_{q \leftarrow i}$ and PDF are given by

$$
\overline{\mathbb{I}}_{i j}\left(z, p_{T}^{\text {veto }}, \mu_{f}\right)=\int_{z}^{1} \frac{d u}{u} \bar{I}_{q \leftarrow i}\left(u, p_{T}^{\text {veto }}, \mu_{f}\right) \bar{I}_{\bar{q} \leftarrow j}\left(z / u, p_{T}^{\text {veto }}, \mu_{f}\right)+(q \leftrightarrow \bar{q}),
$$

and

$$
f f_{i j}\left(y, \mu_{f}\right)=\int_{y}^{1} \frac{d x}{x} f_{i}\left(x, \mu_{f}\right) f_{j}\left(\frac{\tau}{x z}, \mu_{f}\right) .
$$


respectively. Here $(i j)=(q \bar{q}),(q g)$ and $(g \bar{q})$. In order to give precise predictions, we resum the leading singular terms to all orders and include the nonsingular terms, which are suppressed by powers of $\lambda$, up to NLO. Finally, we obtain the RG improved differential cross section as

$$
\frac{d \sigma^{\mathrm{NLO}+\mathrm{NNLL}}\left(p_{T}^{\mathrm{veto}}\right)}{d M^{2}}=\frac{d \sigma^{\mathrm{NNLL}}\left(p_{T}^{\mathrm{veto}}\right)}{d M^{2}}+\left[\frac{d \sigma^{\mathrm{NLO}}}{d M^{2}}-\frac{d \sigma^{\mathrm{NNLL}}\left(p_{T}^{\mathrm{veto}}\right)}{d M^{2}}\right]_{\text {expand to NLO }} .
$$

In this paper our main goal is to derive the factorization expressions and perform the resummation calculations for $H V$ production with a jet veto. The numerical results of the differential NNLO QCD predictions for $H W^{ \pm}$are shown in ref. [12], but their numerical code has not been published. Repeating the complete numerical NNLO QCD calculations is beyond the scope of the this paper. Therefore, we will only include the QCD NLO results in this paper.

The EW gauge boson pair $W^{+} W^{-}$production with a jet veto at the $\mathrm{LHC}$ is a main SM background for the jet veto Higgs boson production channel $g g \rightarrow H \rightarrow W^{+} W^{-}$, and thus it is also significant to perform the resummation calculations for $W^{+} W^{-}$production with a jet veto. Our results can be easily extended to $W^{+} W^{-}$production with a jet veto, and the only differences come from LO cross section and the scale independent terms in the hard functions, which are collected in refs. [49-51].

\section{Numerical results}

In this section, we discuss the numerical results for the $H V$ associated production at the LHC. We choose the following SM input parameters [52]

$$
\begin{aligned}
G_{F} & =1.166379 \times 10^{-5} \mathrm{GeV}^{-2}, \quad m_{H}=125 \mathrm{GeV}, \quad m_{Z}=91.1876 \mathrm{GeV}, \\
m_{W} & =80.398 \mathrm{GeV},
\end{aligned}
$$

and the CKM matrix is given by [52]

$$
V_{\mathrm{CKM}}=\left(\begin{array}{ccc}
0.9751 & 0.2215 & 0.0035 \\
0.2210 & 0.9743 & 0.0410 \\
0 & 0 & 1
\end{array}\right)
$$

Throughout the numerical calculations, we use the MSTW2008 PDF sets and associated strong coupling constant $\alpha_{s}$. In order to resum all logarithmic terms $\ln p_{T}^{\text {veto }} / \mu_{f}$ to all orders, we choose the factorization scale to be $\mu_{f}=p_{T}^{\text {veto }}$ [47]. Besides, the hard matching scale are set as $\mu_{h}^{2}=-M^{2}$ in order to contain the $\pi^{2}$-enhancement effects [53].

\subsection{Leading singular jet vetoed cross section}

For verifying the correctness of the factorization formula in eq. (3.22), we expand the eq. (3.22) to the leading singular terms (black solid line), and compare with the exact NLO results (red dot) calculated by modified Monte Carlo program MCFM [54] in figure 1. We can see that the leading singular terms of the cross section with jet veto can reproduce 

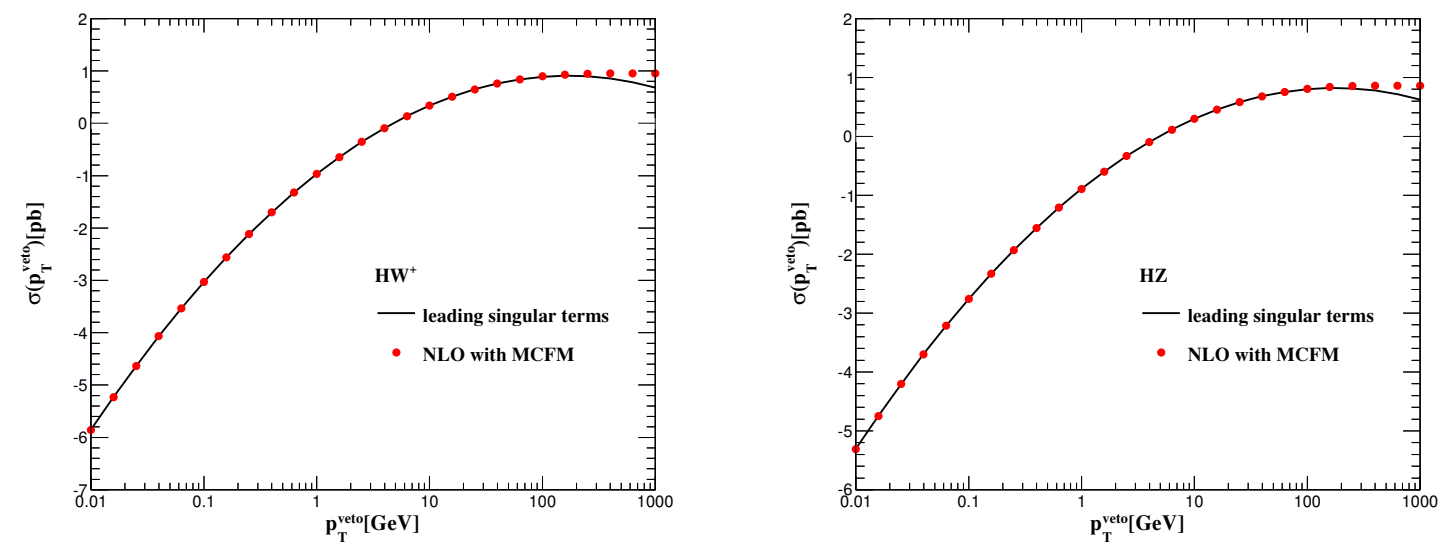

Figure 1. Comparisons of the leading singular and the exact NLO jet vetoed cross sections for $H W^{+}$(left panel) and $H Z$ (right panel) production at the LHC with $\sqrt{S}=14 \mathrm{TeV}$, respectively.
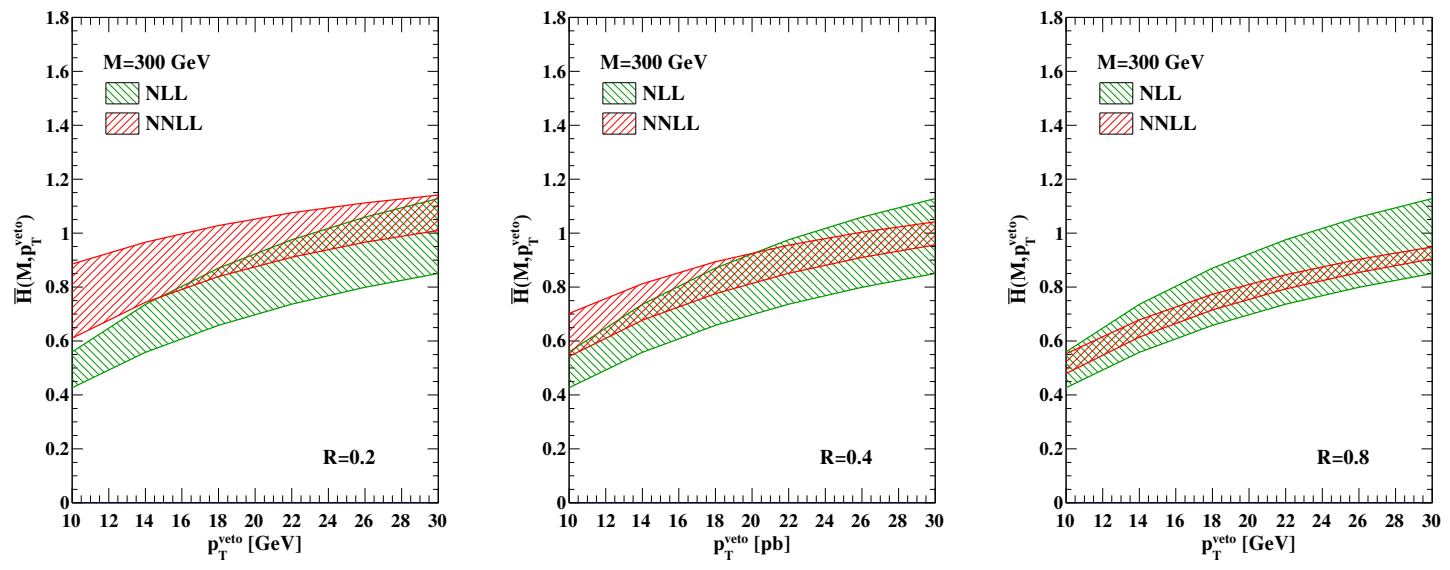

Figure 2. The RG invariant hard function $\bar{H}\left(M, p_{T}^{\text {veto }}\right)$ for three different jet radius parameter $R$, where the bands reflect the scale uncertainties, and $M=300 \mathrm{GeV}$.

the exact NLO jet vetoed cross section in the small $p_{T}^{\text {veto }}$ region. With the increasing of $p_{T}^{\text {veto }}$, the difference between the leading singular and the exact NLO jet veto cross section increases.

\subsection{Scale uncertainties}

In figure 2 we show the scale dependence of $\mathrm{RG}$ invariant hard function $\bar{H}\left(M, p_{T}^{\text {veto }}\right)$ on $p_{T}^{\text {veto }}$ for three different parameters $R$, where the bands reflect the scale uncertainties by varying the scales in the range $p_{T}^{\text {veto }} / 2<\mu_{f}<2 p_{T}^{\text {veto }}$ and $M^{2} / 4<-\mu_{h}^{2}<4 M^{2}$, respectively. In the resummation predictions these two kinds of uncertainties are added in quadrature. From figure 2 we can see that the NLL predictions are independent on the jet radius parameter $R$, while the NNLL predictions strongly depend on $R$. Besides, the NLL and NNLL bands overlap each other, and the scale uncertainties of NNLL results increase as $R$ decreases. When $R=0.8$, the scale uncertainties are significantly reduced from NLL level to NNLL 


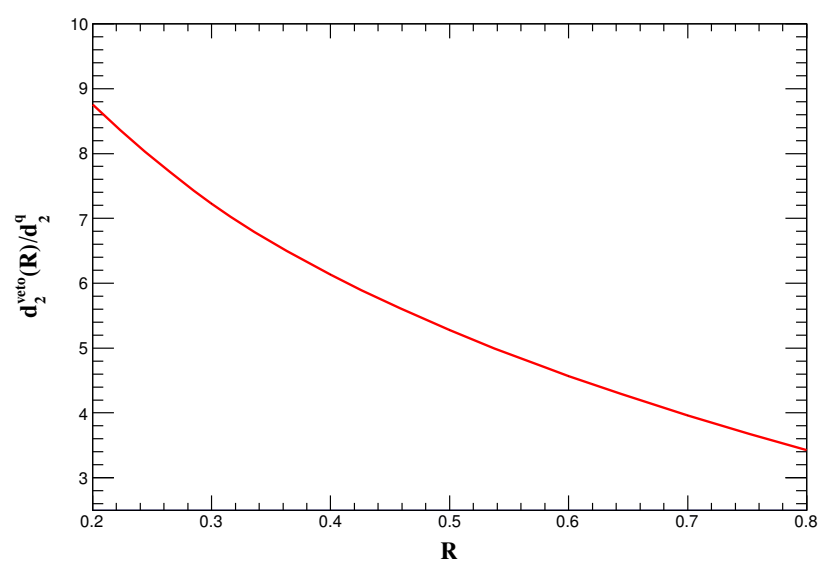

Figure 3. Dependence of the coefficient $d_{2}^{\text {veto }}(R)$ on the jet radius parameter $R$, normalized to $d_{2}^{q}$.

level. And when $R=0.2$, the scale uncertainties are reduced only for large $p_{T}^{\mathrm{veto}}$, and the NNLL and NLL bands overlap only for large $p_{T}^{\text {veto }}$ too. In the small $p_{T}^{\text {veto }}$ region the NNLL bands are broader than the NLL ones, and they are away from each other with the decreasing of $p_{T}^{\text {veto }}$.

In addition to the hard and factorization scale, another scale uncertainty coming from logarithms with collinear anomaly has also been discussed in ref. [35], and it is shown that this uncertainty should not be included in "collinear anomalous" formalism, although this type scale variation can be formalized in an RG framework [37, 38]. Therefore, we apply the same scheme in ref. [35], and also do not consider this kind of uncertainties in our calculations.

At the NNLL level the dependence of the RG invariant hard function $\bar{H}\left(M, p_{T}^{\text {veto }}\right)$ on the jet radius parameter $R$ is caused from the two loop anomaly coefficient $d_{2}^{\text {veto }}(R)$. The $R$ dependence term has the form as

$$
\exp \left[0.54 \frac{d_{2}^{\mathrm{veto}}(R)}{d_{2}^{q}} \alpha_{s}^{2}(\mu) \ln \frac{M}{p_{T}^{\mathrm{veto}}}\right]
$$

where $\alpha_{s}(\mu)$ includes the remaining scale dependence. In order to estimate the scale uncertainties induced by eq. (4.3) at the NNLL level, we show the dependence for the ratio between the coefficient $d_{2}^{\text {veto }}(R)$ and $d_{2}^{q}$ on the jet radius parameter $R$ in figure 3 . With the increasing of the jet radius parameter $R$ from 0.2 to 0.8 , the coefficient $d_{2}^{\text {veto }}(R) / d_{2}^{q}$ rapidly decrease about from 9 to 3 due to the existence of logarithmic terms $\ln R$ in eq. (3.21). Therefore, as shown in figure 2, the remaining scale dependence of RG invariant hard function $\bar{H}\left(M, p_{T}^{\text {veto }}\right)$ increases as the parameter $R$ decreases.

In figure 4 we present NLL (green bands) and NNLL (red bands) resummed predictions on the invariant mass distribution for $H V$ associated production with $p_{T}^{\text {veto }}=30 \mathrm{GeV}$ and $R=0.4$ at the LHC with $\sqrt{S}=14 \mathrm{TeV}$, where the bands reflect the scale uncertainties. We use MSTW2008NLO and MSTW2008NNLO PDF sets for the NLL and NNLL results, respectively. After performing resummation, the theoretical perturbative convergence is 

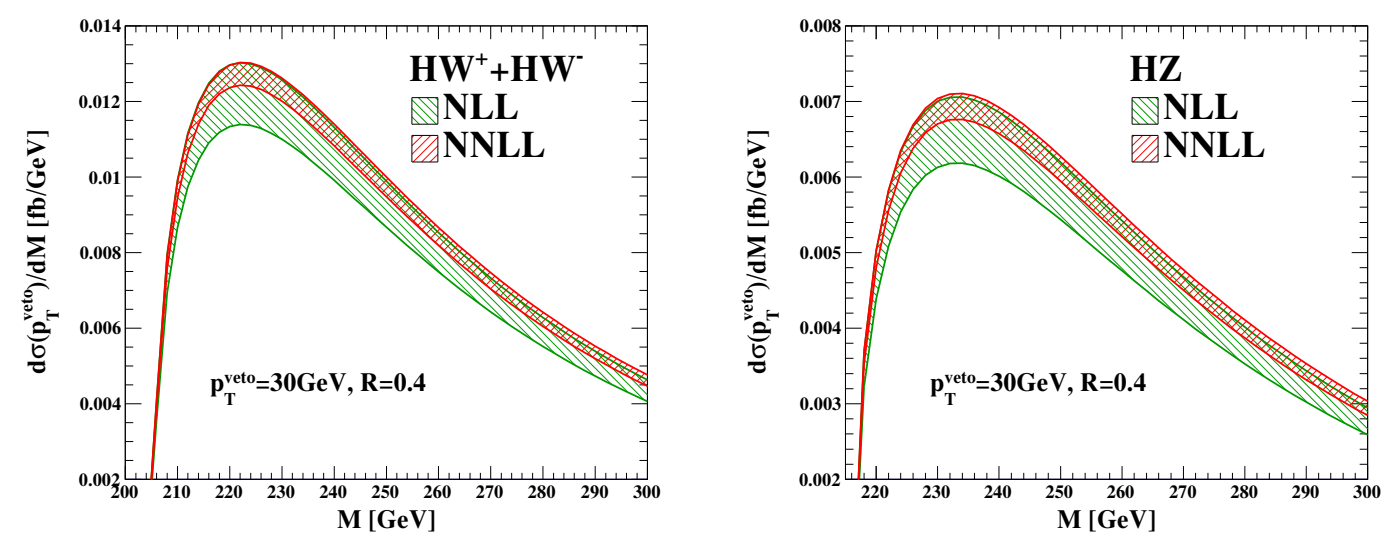

Figure 4. The NLL (green bands) and NNLL (red bands) resummed invariant mass distributions for $H W^{ \pm}$(left panel) and $H Z$ (right panel) associated production with $p_{T}^{\text {veto }}=20 \mathrm{GeV}$ and $R=0.4$ at the LHC with $\sqrt{S}=14 \mathrm{TeV}$, where the bands reflect the scale uncertainties.

well behaved, and the scale uncertainties are reduced from NLL level to NNLL level for all the invariant mass region.

In figure 5, we show the scale dependence of the NLL (green bands) and NNLL (red bands) resummed jet veto cross section on $p_{T}^{\text {veto }}$ at the LHC with $\sqrt{S}=14 \mathrm{TeV}$ for three different parameters $R=0.2,0.4$ and 0.8 , where the bands reflect the scale uncertainties. In the case of $H W^{ \pm}$production, the resummed jet veto cross section at the NLL level is independent on the radius parameter $R$, and the scale uncertainties are about $13 \%$. Similar to the case of RG invariant hard function, with the decreasing of the parameter $R$, the scale uncertainties of NNLL results increase. When $R=0.8,0.4$ and 0.2 , the scale uncertainties at the NNLL level are reduced to $2 \%, 5 \%$ and $8 \%$ for $p_{T}^{\text {veto }}=35$, and $7 \%, 10 \%$ and $17 \%$ for $p_{T}^{\text {veto }}=10 \mathrm{GeV}$, respectively. Obviously, the scale uncertainties are reduced when $R=0.8$. Besides, in the large $p_{T}^{\text {veto }}$ region the scale uncertainties are also reduced and the NNLL and NLL bands also overlap when $R=0.2$ and 0.4 . However, in the small $p_{T}^{\text {veto }}$ region the NNLL uncertainties are larger than the NLL ones, and NNLL and NLL bands are away from each other. The origin of these $R$ dependence is also caused from eq. (4.3).

\subsection{RG improved phenomenology predictions at the LHC}

$H V$ associated production is an important process to study the Higgs boson at the LHC. Both of two decay modes, $h \rightarrow b \bar{b}$ and $h \rightarrow W^{+} W^{-}$have been searched by the ALTAS [55, 56] and CMS [57, 58] collaborations, respectively. The results from ATLAS show that no significant excess is observed over the SM expectations, with or without a $m_{H}=125 \mathrm{GeV}$ Higgs boson. And the results from CMS show that a small excess above the SM background expectation is found. Since there does not exit enough $H V$ events produced at the LHC, the corresponding jet veto studies can not be completed. With the increasing of the luminosity, $H V$ production will be more important to study the property of the SM Higgs boson, and the studies about jet veto for this process will also be attracted more attentions from experimentalists. 

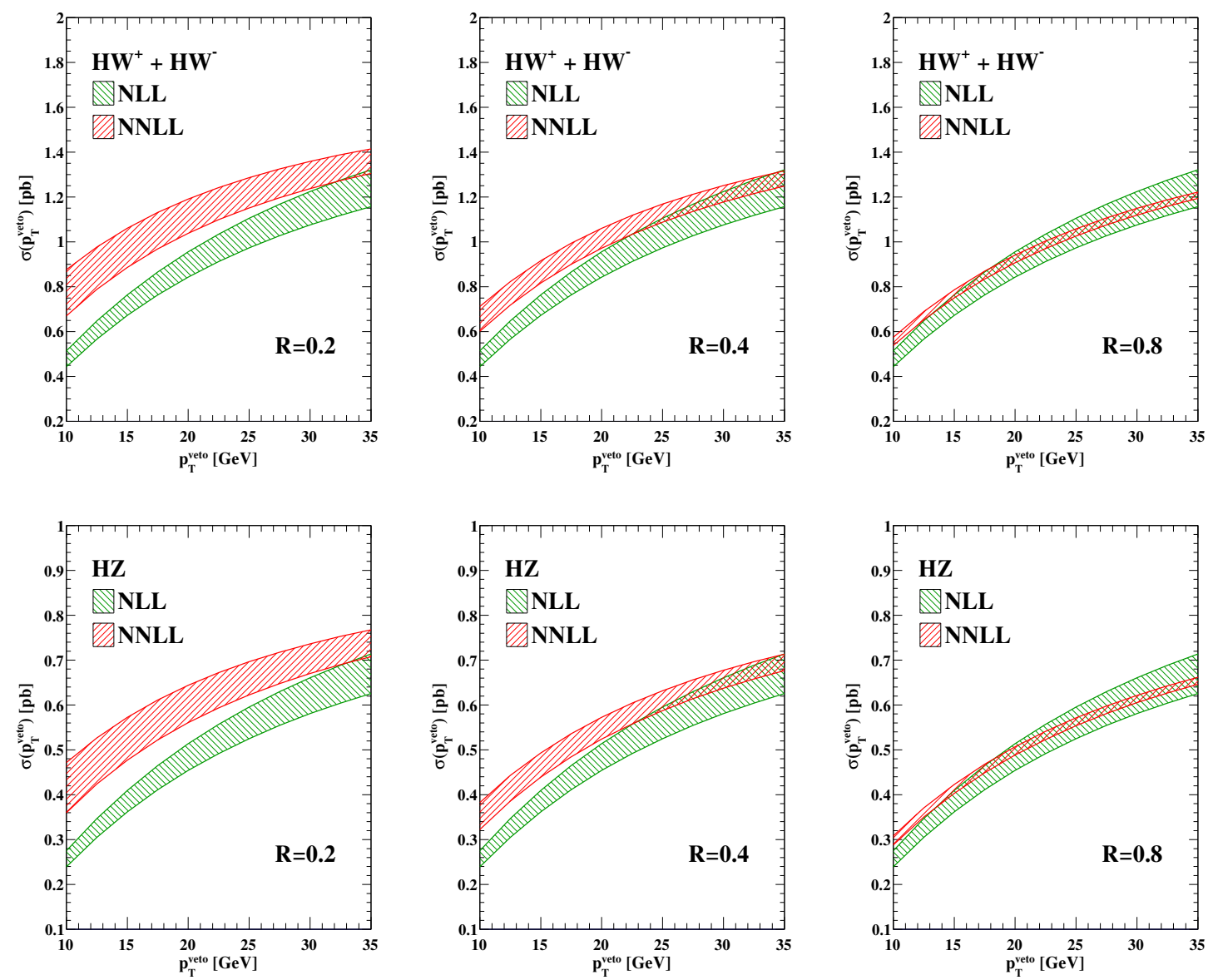

Figure 5. The NLL (green bands) and NNLL (red bands) resummed jet veto cross section for $H V$ associated production at the LHC with $\sqrt{S}=14 \mathrm{TeV}$ for three different jet radius parameter $R=0.2,0.4$ and 0.8 , where the bands reflect the scale uncertainties.

In figure 6 we present the NLO+NNLL and NLO jet vetoed invariant mass distribution for $H V$ associated production at the LHC with $\sqrt{S}=14 \mathrm{TeV}$, where $p_{T}^{\text {veto }}=20 \mathrm{GeV}$ and $R=0.4$ are chosen. The bands represent the scale uncertainties. We present the NLO results in two benchmark schemes, $\mu_{f} \sim M$ (red bands) and $\mu_{f} \sim p_{T}^{\text {veto }}$ (green bands), respectively. Compared to NLO+NNLL results (black bands), for $\mu_{f} \sim M$ the NLO predictions are similar to the NLO+NNLL ones, but suffer from large scale uncertainties in all the invariant mass region. However, when $\mu_{f} \sim p_{T}^{\text {veto }}$, the NLO predictions have large scale uncertainties only in the large invariant mass region, but underestimate the theoretical prediction in all the invariant mass region.

After performing the integration over the invariant mass, we can get the jet vetoed cross sections. In figure 7 we present the NLO+NNLL jet vetoed cross section at the $14 \mathrm{TeV}$ LHC for $R=0.2,0.4$ and 0.8 , where the bands reflect the scale uncertainties. It is shown that the NLO+NNLL predictions strongly depend on the jet radius parameter $R$. With the increasing of $R$ value, the NLO+NNLL predictions decrease and the scale uncertainties reduce. 

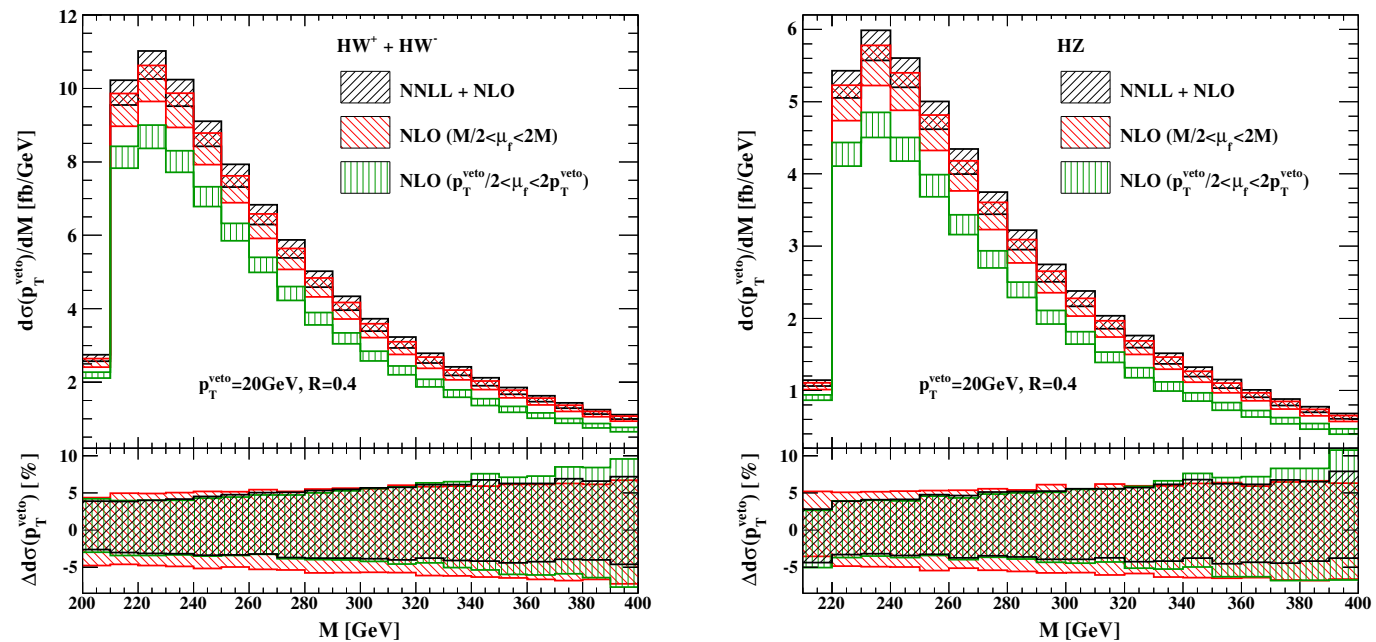

Figure 6. The NLO and NLO+NNLL jet vetoed predictions for $H V$ associated production invariant mass distribution with $p_{T}^{\text {veto }}=20 \mathrm{GeV}$ and $R=0.4$ at the LHC with $\sqrt{S}=14 \mathrm{TeV}$, where the bands reflect the scale uncertainties.
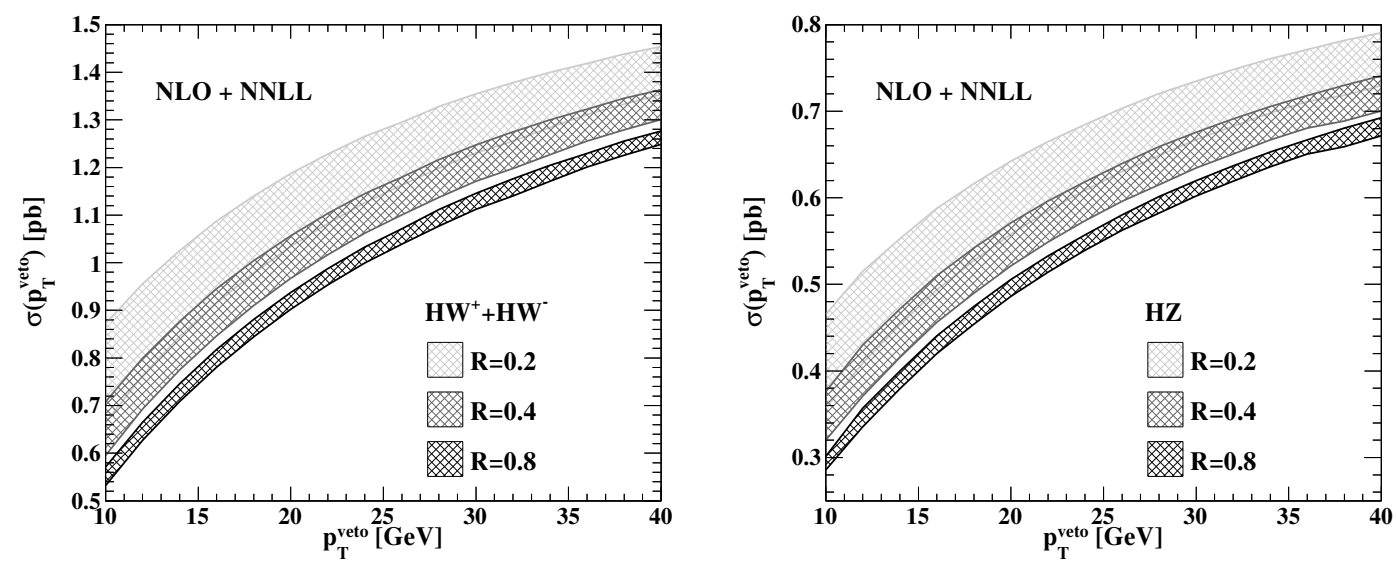

Figure 7. The NLO+NNLL predictions for $H V$ associated production cross section with a jet veto at the $14 \mathrm{TeV}$ LHC for $R=0.2,0.4$ and 0.8 , where the bands reflect the scale uncertainties.

In figure 8, we present the NLO and NLO+NNLL predictions for jet vetoed cross section at the $14 \mathrm{TeV}$ LHC, where the bands reflect the scale uncertainties. In the NLO+NNLL predictions the jet radius parameters $R$ are chosen as $R=0.4$ (blue bands) and 0.5 (black bands), respectively. Besides, the NLO results are presented in two benchmark schemes, $\mu_{f} \sim M$ (red bands) and $\mu_{f} \sim p_{T}^{\text {veto }}$ (green bands), respectively. Obviously, the NLO results suffer from much larger scale uncertainties than the NLO+NNLL predictions in the small $p_{T}^{\text {veto }}$ region. Especially, when $\mu_{f} \sim p_{T}^{\text {veto }}$ is chosen, the NLO predictions break down in the small $p_{T}^{\text {veto }}$ region, while after including resummation effects the theoretical convergence are well behaved.

In table 1 and table 2 we list the NLO+NNLL jet vetoed cross section at the LHC with $\sqrt{S}=13$ and $14 \mathrm{TeV}$, respectively. Here, besides scale uncertainties are taken into account, to estimate the PDF uncertainties, we use the MSTW2008 90\% C.L. PDF sets [59], which 

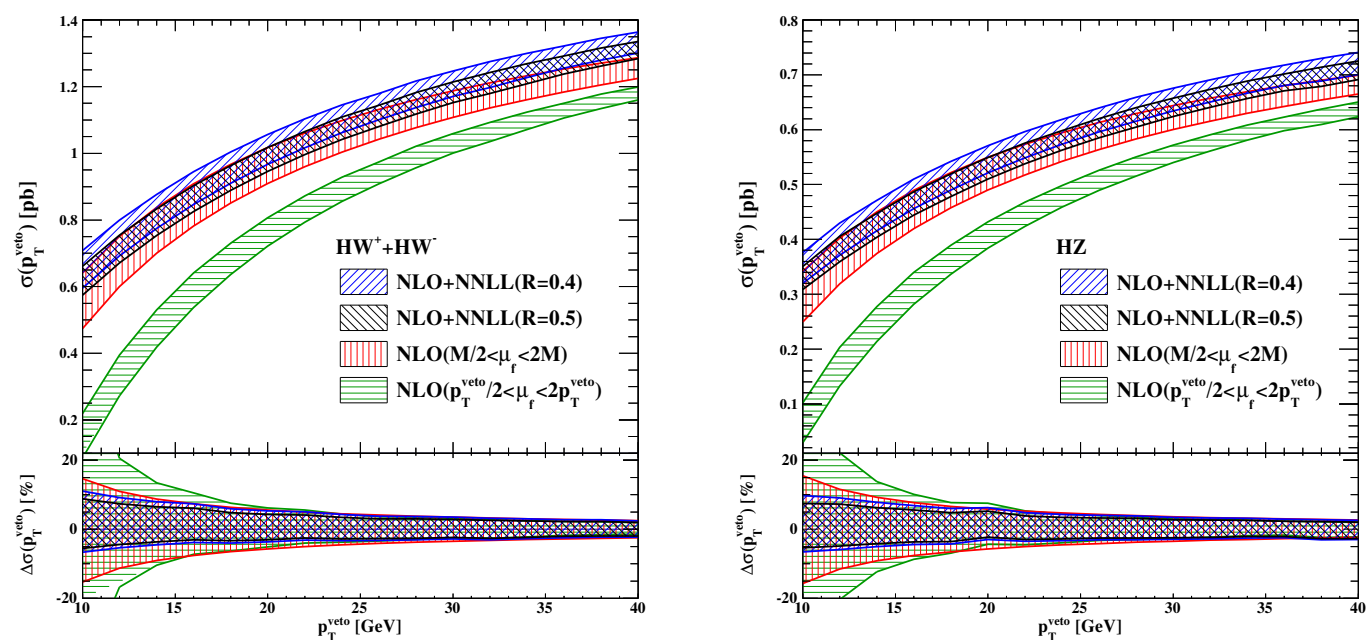

Figure 8. The NLO and NLO+NNLL predictions for jet vetoed cross section at the $14 \mathrm{TeV}$ LHC, where the bands reflect the scale uncertainties.

\begin{tabular}{|lcccccccc|}
\hline & \multicolumn{3}{c}{$R=0.4$} & & \multicolumn{3}{c|}{$R=0.5$} \\
\cline { 2 - 5 } \cline { 7 - 8 }$p_{T}^{\text {veto }}[\mathrm{GeV}]$ & 20 & 25 & 30 & & 20 & 25 & 30 \\
\hline$\sigma^{H W}[\mathrm{pb}]$ & 0.92 & 1.03 & 1.10 & & 0.90 & 1.00 & 1.08 \\
Scale $[\%]$ & $+5.1-3.7$ & $+4.3-3.2$ & $+3.5-2.8$ & & $+4.1-3.1$ & $+3.5-2.7$ & $+2.8-2.3$ \\
PDF $[\%]$ & $+4.0-3.6$ & $+3.9-3.5$ & $+3.8-3.4$ & & $+4.0-3.6$ & $+3.9-3.5$ & $+3.8-3.4$ \\
\hline$\sigma^{H Z}[\mathrm{pb}]$ & 0.498 & 0.554 & 0.598 & & 0.484 & 0.541 & 0.585 \\
Scale $[\%]$ & $+5.5-3.9$ & $+4.3-3.4$ & $+3.6-2.9$ & & $+4.5-3.3$ & $+3.5-2.9$ & $+2.9-2.5$ \\
PDF $[\%]$ & $+4.0-3.5$ & $+3.9-3.3$ & $+3.8-3.3$ & & $+4.0-3.5$ & $+3.9-3.3$ & $+3.8-3.3$ \\
\hline
\end{tabular}

Table 1. The jet vetoed cross section at the $13 \mathrm{TeV}$ LHC with jet radius parameter $R=0.4$ and 0.5 , respectively.

are known to provide very close results to the PDF4LHC working group recommendation for the envelop prescription [60]. Table 1 and table 2 show that the scale and PDF uncertainties are almost same order. Moreover, with the increasing of the $p_{T}^{\mathrm{veto}}$ and $R$, the scale uncertainties decrease, while the PDF uncertainties almost do not change.

\section{Conclusion}

We have studied the resummation effects for the $H V$ associated production at the LHC with a jet veto in SCET using "collinear anomalous" formalism. We calculate the jet vetoed invariant mass distribution and the cross section for this process at Next-to-Next-toLeading-Logarithmic level, which are matched to the QCD Next-to-Leading Order results, and compare the differences of the resummation effects with different jet veto $p_{T}^{\text {veto }}$ and jet radius $R$. Our results show that both resummation enhancement effects and the scale uncertainties decrease with the increasing of jet veto $p_{T}^{\text {veto }}$ and jet radius $R$, respectively. 


\begin{tabular}{|lcccccccc|}
\hline & \multicolumn{3}{c}{$R=0.4$} & & \multicolumn{3}{c|}{$R=0.5$} \\
\cline { 2 - 3 } \cline { 6 - 8 }$p_{T}^{\text {veto }}[\mathrm{GeV}]$ & 20 & 25 & 30 & & 20 & 25 & 30 \\
\hline$\sigma^{H W}[\mathrm{pb}]$ & 1.00 & 1.12 & 1.20 & & 0.98 & 1.08 & 1.17 \\
Scale [\%] & $+5.3-3.6$ & $+4.4-3.0$ & $+3.5-2.8$ & & $+4.3-3.0$ & $+3.6-2.5$ & $+2.9-2.4$ \\
PDF [\%] & $+3.9-3.5$ & $+3.9-3.5$ & $+3.8-3.4$ & & $+3.9-3.5$ & $+3.9-3.5$ & $+3.8-3.4$ \\
\hline$\sigma^{H Z}[\mathrm{pb}]$ & 0.537 & 0.604 & 0.653 & & 0.522 & 0.591 & 0.640 \\
Scale $[\%]$ & $+6.3-2.9$ & $+4.0-3.2$ & $+3.4-2.9$ & & $+5.3-2.3$ & $+3.2-2.7$ & $+2.8-2.5$ \\
PDF [\%] & $+4.0-3.4$ & $+3.8-3.3$ & $+3.7-3.2$ & & $+4.0-3.4$ & $+3.8-3.3$ & $+3.7-3.2$ \\
\hline
\end{tabular}

Table 2. The jet vetoed cross section at the $14 \mathrm{TeV}$ LHC with jet radius parameter $R=0.4$ and 0.5 , respectively.
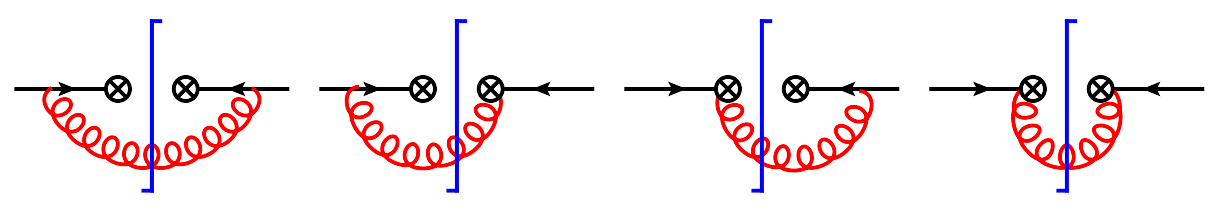

Figure 9. Feynman diagrams contribution to the NLO beam function $\mathcal{I}_{q \leftarrow q}$.

When $p_{T}^{\text {veto }}=25 \mathrm{GeV}$ and $R=0.4(0.5)$, the resummation effects reduce the scale uncertainties of the Next-to-Leading Order jet vetoed cross sections to about $7 \%$ (6\%), which lead to increased confidence on the theoretical predictions. Besides, after including resummation effects, the PDF uncertainties of jet vetoed cross section are about 7\%. Our results can help to precisely study the physical property of the SM Higgs boson through Higgs and vector boson associated production at the LHC in the future.

\section{Acknowledgments}

We would like to thank Hua Xing Zhu for helpful discussions. This work was supported in part by the National Natural Science Foundation of China under Grants NO. 11375013 and NO. 11135003.

\section{A Calculation of beam functions}

In this appendix we show the details of calculating the beam functions. At the NLO, the beam functions receive the contributions from the diagrams shown in figure 9 and we have the sum of these diagrams,

$$
\begin{aligned}
\mathcal{I}_{q \leftarrow q}^{(1), \text { bare }}\left(z, p_{T}^{\text {veto }}, \mu\right)=g_{s}^{2} C_{F} \mu^{2 \epsilon} \int \frac{d^{D} k}{(2 \pi)^{D-1}} & \left(\frac{\nu}{k^{+}}\right)^{\alpha} \delta\left(k^{2}\right) \theta\left(k^{0}\right) \delta\left(k^{-}-(1-z) p^{-}\right) \\
& \times \theta\left(p_{T}^{\text {veto }}-k_{T}\right) \frac{k^{-}}{k_{T}^{2}}\left[(D-2)(1-z)+\frac{4 z}{1-z}\right],
\end{aligned}
$$




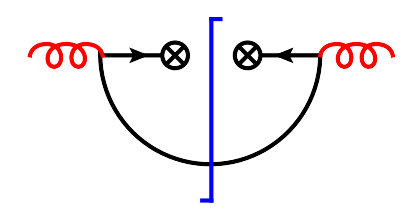

Figure 10. Feynman diagram contributions to the NLO beam function $\mathcal{I}_{q \leftarrow g}$.

where we have suppressed the $\overline{\mathrm{MS}}$ factor $\left(e^{\gamma_{\mathrm{E}}} / 4 \pi\right)^{\epsilon}$ and the analytic regularization method of ref. [45] is used. The integration measure can be written as

$$
\begin{aligned}
& d^{D} k \delta\left(k^{2}\right) \theta\left(k^{0}\right) \delta\left(k^{-}-(1-z) p^{-}\right) \theta\left(p_{T}^{\text {veto }}-k_{T}\right) \\
& =\frac{1}{2 p^{-}} \frac{1}{1-z} \frac{2 \pi^{1 / 2-\epsilon}}{\Gamma(1 / 2-\epsilon)} \int_{0}^{p_{T}^{\text {veto }}} d k_{T} d \theta k_{T}^{1-2 \epsilon} \sin ^{-2 \epsilon} \theta
\end{aligned}
$$

Thus, we have bare $\mathcal{I}_{q \leftarrow q}$ up to NLO,

$$
\begin{aligned}
& \mathcal{I}_{q \leftarrow q}^{\text {bare }}(z,\left.p_{T}^{\text {veto }}, \mu\right)=\delta(1-z)-\frac{C_{F} \alpha_{s}}{2 \pi}\left\{\delta(1-z)\left(-\frac{2}{\epsilon^{2}}+L_{\perp}^{2}+\frac{\pi^{2}}{6}\right)\right. \\
&\left.+\left(\frac{1}{\epsilon}+L_{\perp}\right)\left[\left(\frac{2}{\alpha}-2 \ln \frac{\mu^{2}}{\nu p_{1}^{-}}\right) \delta(1-z)+\frac{2}{(1-z)_{+}}-z-1\right]-(1-z)\right\} .
\end{aligned}
$$

Similarly, the bare $\mathcal{I}_{\bar{q} \leftarrow \bar{q}}$ is given by

$$
\begin{aligned}
\mathcal{I}_{\bar{q} \leftarrow \bar{q}}^{\text {bare }}\left(z, p_{T}^{\text {veto }}, \mu\right)=\delta(1-z)-\frac{C_{F} \alpha_{s}}{2 \pi}\left\{\left(\frac{1}{\epsilon}+L_{\perp}\right)\right. & {\left[\left(-\frac{2}{\alpha}-2 \ln \frac{\nu}{p_{2}^{+}}\right) \delta(1-z)\right.} \\
& \left.\left.+\frac{2}{(1-z)_{+}}-z-1\right]-(1-z)\right\} .
\end{aligned}
$$

The product of two beam functions is independent on the regulator $\alpha$ and well defined in the general dimensional regularization.

The evaluation of the beam function $\mathcal{I}_{q \leftarrow g}$ is independent on the regulator $\alpha$, and the corresponding Feynman diagram is shown in figure 10. After performing analytical calculation, we have

$$
\mathcal{I}_{q \leftarrow g}^{\text {bare }}\left(z, p_{T}^{\text {veto }}, \mu\right)=-\frac{T_{F} \alpha_{s}}{2 \pi}\left\{\left(\frac{1}{\epsilon}+L_{\perp}\right)\left[z^{2}+(1-z)^{2}\right]-2 z(1-z)\right\} .
$$

By means of eq. (A.4) and (A.5), after $\overline{\mathrm{MS}}$ subtraction we can exact the coefficients $d_{1}^{\mathrm{veto}}(R)$, $R_{q \leftarrow q}(z)$ and $R_{q \leftarrow g}(z)$, directly.

Open Access. This article is distributed under the terms of the Creative Commons Attribution License (CC-BY 4.0), which permits any use, distribution and reproduction in any medium, provided the original author(s) and source are credited. 


\section{References}

[1] ATLAS collaboration, Observation of a new particle in the search for the standard model Higgs boson with the ATLAS detector at the LHC, Phys. Lett. B 716 (2012) 1 [arXiv: 1207.7214] [INSPIRE].

[2] CMS collaboration, Observation of a new boson at a mass of $125 \mathrm{GeV}$ with the CMS experiment at the LHC, Phys. Lett. B 716 (2012) 30 [arXiv:1207.7235] [INSPIRE].

[3] T. Han and S. Willenbrock, QCD correction to the $p p \rightarrow W H$ and $Z H$ total cross-sections, Phys. Lett. B 273 (1991) 167 [INSPIRE].

[4] H. Baer, B. Bailey and J. Owens, $O\left(\alpha_{s}\right)$ Monte Carlo approach to $W+$ Higgs associated production at hadron supercolliders, Phys. Rev. D 47 (1993) 2730 [InSPIRE].

[5] J. Ohnemus and W.J. Stirling, Order $\alpha_{s}$ corrections to the differential cross-section for the WH intermediate mass Higgs signal, Phys. Rev. D 47 (1993) 2722 [INSPIRE].

[6] B.A. Kniehl, Associated production of Higgs and $Z$ bosons from gluon fusion in hadron collisions, Phys. Rev. D 42 (1990) 2253 [INSPIRE].

[7] M. Ciccolini, S. Dittmaier and M. Krämer, Electroweak radiative corrections to associated WH and ZH production at hadron colliders, Phys. Rev. D 68 (2003) 073003 [hep-ph/0306234] [INSPIRE].

[8] R. Hamberg, W. van Neerven and T. Matsuura, A complete calculation of the order $\alpha_{s}^{2}$ correction to the Drell-Yan K factor, Nucl. Phys. B 359 (1991) 343 [Erratum ibid. B 644 (2002) 403-404] [INSPIRE].

[9] R.V. Harlander and W.B. Kilgore, Next-to-next-to-leading order Higgs production at hadron colliders, Phys. Rev. Lett. 88 (2002) 201801 [hep-ph/0201206] [INSPIRE].

[10] O. Brein, A. Djouadi and R. Harlander, NNLO QCD corrections to the Higgs-strahlung processes at hadron colliders, Phys. Lett. B 579 (2004) 149 [hep-ph/0307206] [INSPIRE].

[11] O. Brein, R.V. Harlander and T.J. Zirke, vh@nnlo — Higgs Strahlung at hadron colliders, Comput. Phys. Commun. 184 (2013) 998 [arXiv:1210.5347] [InSPIRE].

[12] G. Ferrera, M. Grazzini and F. Tramontano, Associated WH production at hadron colliders: a fully exclusive QCD calculation at NNLO, Phys. Rev. Lett. 107 (2011) 152003 [arXiv:1107.1164] [INSPIRE].

[13] S. Catani and M. Grazzini, An NNLO subtraction formalism in hadron collisions and its application to Higgs boson production at the LHC, Phys. Rev. Lett. 98 (2007) 222002 [hep-ph/0703012] [INSPIRE].

[14] A. Banfi and J. Cancino, Implications of QCD radiative corrections on high-p T Higgs searches, Phys. Lett. B 718 (2012) 499 [arXiv:1207.0674] [INSPIRE].

[15] S. Dawson, T. Han, W.K. Lai, A.K. Leibovich and I. Lewis, Resummation effects in vector-boson and Higgs associated production, Phys. Rev. D 86 (2012) 074007 [arXiv: 1207 .4207] [INSPIRE].

[16] C. Anastasiou, G. Dissertori, F. Stockli and B.R. Webber, QCD radiation effects on the

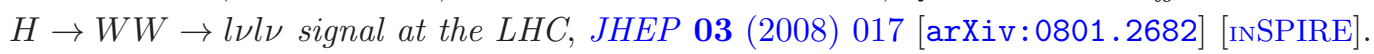

[17] C. Anastasiou, G. Dissertori, M. Grazzini, F. Stockli and B.R. Webber, Perturbative QCD

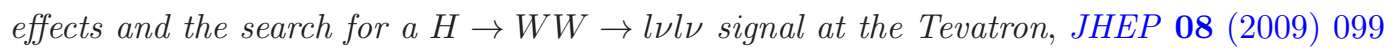
[arXiv:0905.3529] [INSPIRE]. 
[18] I.W. Stewart, F.J. Tackmann and W.J. Waalewijn, Factorization at the LHC: from PDFs to initial state jets, Phys. Rev. D 81 (2010) 094035 [arXiv:0910.0467] [INSPIRE].

[19] I.W. Stewart, F.J. Tackmann and W.J. Waalewijn, N-jettiness: an inclusive event shape to veto jets, Phys. Rev. Lett. 105 (2010) 092002 [arXiv: 1004.2489] [INSPIRE].

[20] C.F. Berger, C. Marcantonini, I.W. Stewart, F.J. Tackmann and W.J. Waalewijn, Higgs production with a central jet veto at NNLL+NNLO, JHEP 04 (2011) 092 [arXiv: 1012.4480] [INSPIRE].

[21] I.W. Stewart, F.J. Tackmann and W.J. Waalewijn, The beam thrust cross section for Drell-Yan at NNLL order, Phys. Rev. Lett. 106 (2011) 032001 [arXiv:1005.4060] [INSPIRE].

[22] A. Papaefstathiou, J.M. Smillie and B.R. Webber, Resummation of transverse energy in vector boson and Higgs boson production at hadron colliders, JHEP 04 (2010) 084 [arXiv: 1002 .4375] [INSPIRE].

[23] F.J. Tackmann, J.R. Walsh and S. Zuberi, Resummation properties of jet vetoes at the LHC, Phys. Rev. D 86 (2012) 053011 [arXiv:1206.4312] [INSPIRE].

[24] A. Banfi, G.P. Salam and G. Zanderighi, Principles of general final-state resummation and automated implementation, JHEP 03 (2005) 073 [hep-ph/0407286] [INSPIRE].

[25] A. Banfi, G.P. Salam and G. Zanderighi, $N L L+N N L O$ predictions for jet-veto efficiencies in Higgs-boson and Drell-Yan production, JHEP 06 (2012) 159 [arXiv:1203.5773] [INSPIRE].

[26] C.W. Bauer, S. Fleming, D. Pirjol and I.W. Stewart, An effective field theory for collinear and soft gluons: heavy to light decays, Phys. Rev. D 63 (2001) 114020 [hep-ph/0011336] [INSPIRE].

[27] C.W. Bauer, D. Pirjol and I.W. Stewart, Soft collinear factorization in effective field theory, Phys. Rev. D 65 (2002) 054022 [hep-ph/0109045] [INSPIRE].

[28] M. Beneke, A. Chapovsky, M. Diehl and T. Feldmann, Soft collinear effective theory and heavy to light currents beyond leading power, Nucl. Phys. B 643 (2002) 431 [hep-ph/0206152] [INSPIRE].

[29] T. Becher and M. Neubert, Drell-Yan production at small $q_{T}$, transverse parton distributions and the collinear anomaly, Eur. Phys. J. C 71 (2011) 1665 [arXiv:1007.4005] [InSPIRE].

[30] T. Becher and M. Neubert, Factorization and NNLL resummation for Higgs production with a jet veto, JHEP 07 (2012) 108 [arXiv:1205.3806] [INSPIRE].

[31] A. Banfi, P.F. Monni, G.P. Salam and G. Zanderighi, Higgs and Z-boson production with a jet veto, Phys. Rev. Lett. 109 (2012) 202001 [arXiv:1206.4998] [INSPIRE].

[32] G. Bozzi, S. Catani, D. de Florian and M. Grazzini, The $q_{T}$ spectrum of the Higgs boson at the LHC in QCD perturbation theory, Phys. Lett. B 564 (2003) 65 [hep-ph/0302104] [INSPIRE].

[33] G. Bozzi, S. Catani, D. de Florian and M. Grazzini, Transverse-momentum resummation and the spectrum of the Higgs boson at the LHC, Nucl. Phys. B 737 (2006) 73 [hep-ph/0508068] [INSPIRE].

[34] G. Bozzi, S. Catani, G. Ferrera, D. de Florian and M. Grazzini, Production of Drell-Yan lepton pairs in hadron collisions: Transverse-momentum resummation at next-to-next-to-leading logarithmic accuracy, Phys. Lett. B 696 (2011) 207 [arXiv: 1007.2351] [INSPIRE]. 
[35] T. Becher, M. Neubert and L. Rothen, Factorization and $N^{3} L L_{p}+N N L O$ predictions for the Higgs cross section with a jet veto, JHEP 10 (2013) 125 [arXiv:1307.0025] [INSPIRE].

[36] I.W. Stewart, F.J. Tackmann, J.R. Walsh and S. Zuberi, Jet $p_{T}$ resummation in Higgs Production at $N N L L^{\prime}+N N L O$, arXiv:1307.1808 [INSPIRE].

[37] J.-y. Chiu, A. Jain, D. Neill and I.Z. Rothstein, The rapidity renormalization group, Phys. Rev. Lett. 108 (2012) 151601 [arXiv:1104.0881] [InSPIRE].

[38] J.-Y. Chiu, A. Jain, D. Neill and I.Z. Rothstein, A formalism for the systematic treatment of rapidity logarithms in quantum field theory, JHEP 05 (2012) 084 [arXiv: 1202.0814] [INSPIRE].

[39] G.P. Salam, Towards jetography, Eur. Phys. J. C 67 (2010) 637 [arXiv:0906.1833] [INSPIRE].

[40] S. Catani, Y.L. Dokshitzer, M. Seymour and B. Webber, Longitudinally invariant $K_{t}$ clustering algorithms for hadron hadron collisions, Nucl. Phys. B 406 (1993) 187 [InSPIRE].

[41] S.D. Ellis and D.E. Soper, Successive combination jet algorithm for hadron collisions, Phys. Rev. D 48 (1993) 3160 [hep-ph/9305266] [INSPIRE].

[42] Y.L. Dokshitzer, G. Leder, S. Moretti and B. Webber, Better jet clustering algorithms, JHEP 08 (1997) 001 [hep-ph/9707323] [INSPIRE].

[43] M. Wobisch and T. Wengler, Hadronization corrections to jet cross-sections in deep inelastic scattering, hep-ph/9907280 [INSPIRE].

[44] M. Cacciari, G.P. Salam and G. Soyez, The anti-k $k_{t}$ jet clustering algorithm, JHEP 04 (2008) 063 [arXiv:0802.1189] [INSPIRE].

[45] T. Becher and G. Bell, Analytic regularization in soft-collinear effective theory, Phys. Lett. B 713 (2012) 41 [arXiv:1112.3907] [INSPIRE].

[46] M.G. Echevarria, A. Idilbi and I. Scimemi, Definition and evolution of transverse momentum distributions, Int. J. Mod. Phys. Conf. Ser. 20 (2012) 92.

[47] T. Becher, M. Neubert and G. Xu, Dynamical threshold enhancement and resummation in Drell-Yan production, JHEP 07 (2008) 030 [arXiv:0710.0680] [INSPIRE].

[48] V. Ahrens, M. Neubert and L. Vernazza, Structure of infrared singularities of gauge-theory amplitudes at three and four loops, JHEP 09 (2012) 138 [arXiv:1208.4847] [INSPIRE].

[49] S. Frixione, A next-to-leading order calculation of the cross-section for the production of $W^{+} W^{-}$pairs in hadronic collisions, Nucl. Phys. B 410 (1993) 280 [INSPIRE].

[50] S. Dawson, I.M. Lewis and M. Zeng, Threshold resummed and approximate NNLO results for $W^{+} W^{-}$pair production at the LHC, Phys. Rev. D 88 (2013) 054028 [arXiv:1307.3249] [INSPIRE].

[51] Y. Wang, C.S. Li, Z.L. Liu, D.Y. Shao and H.T. Li, Transverse-momentum resummation for gauge boson pair production at the hadron collider, Phys. Rev. D 88 (2013) 114017 [arXiv: 1307.7520] [INSPIRE].

[52] Particle Data Group collaboration, J. Beringer et al., Review of particle physics, Phys. Rev. D 86 (2012) 010001 [inSPIRE].

[53] V. Ahrens, T. Becher, M. Neubert and L.L. Yang, Origin of the large perturbative corrections to Higgs production at hadron colliders, Phys. Rev. D 79 (2009) 033013 [arXiv:0808.3008] [INSPIRE]. 
[54] J.M. Campbell and R.K. Ellis. MCFM for the Tevatron and the LHC, Nucl. Phys. Proc. Suppl. 205-206 (2010) 10.

[55] ATLAS collaboration, Search for associated production of the Higgs boson in the $W H \rightarrow W W W(*) \rightarrow l \nu l \nu l \nu$ and $Z H \rightarrow Z W W(*) \rightarrow l l l \nu l \nu$ channels with the ATLAS detector at the LHC, ATLAS-CONF-2013-075 (2013).

[56] ATLAS collaboration, Search for the bb decay of the standard model Higgs boson in associated W/ZH production with the ATLAS detector, ATLAS-CONF-2013-079 (2013).

[57] CMS collaboration, VH with $H \rightarrow W W \rightarrow \ell \nu \ell \nu$ and $V \rightarrow j j$, CMS-PAS-HIG-13-017 (2013).

[58] CMS collabration, Search for the standard model Higgs boson produced in association with a $W$ or a $Z$ boson and decaying to bottom quarks, Phys. Rev. D 89 (2014) 012003 [arXiv: 1310.3687] [INSPIRE].

[59] A.D. Martin, W.J. Stirling, R.S. Thorne and G. Watt, Uncertainties on $\alpha_{s}$ in global PDF analyses and implications for predicted hadronic cross sections, Eur. Phys. J. C 64 (2009) 653 [arXiv:0905.3531] [INSPIRE].

[60] M. Botje et al., The PDF 4 LHC working group interim recommendations, arXiv:1101.0538 [INSPIRE]. 